\title{
Constant-pH MD simulations of DMPA/DMPC lipid bilayers
}

\author{
Hugo A. F. Santos, ${ }^{\dagger}$ Diogo Vila-Viçosa, ${ }^{\ddagger}$ Vitor H. Teixeira, ${ }^{\ddagger}$ António M.
} Baptista, " and Miguel Machuqueiro*,

†BioISI-Biosystems $\&$ Integrative Sciences Institute, Faculdade de Ciências, Universidade de Lisboa, 1749-016 Lisboa, Portugal

$\ddagger$ Centro de Química e Bioquímica and Departamento de Química e Bioquímica, Faculdade de Ciências, Universidade de Lisboa, 1749-016 Lisboa, Portugal

『Instituto de Tecnologia Química e Biológica António Xavier, Universidade Nova de Lisboa, Av. da República, 2780-157 Oeiras, Portugal

E-mail: machuque@ciencias.ulisboa.pt

Phone: +351-21-7500112. Fax: +351-21-7500088

Supporting Information 
Table S1. Pre-equilibrated lipid bilayers of pure DMPC at several ionizations. DMPC ${ }^{+1}$ refers to the protonated form of DMPC.

\begin{tabular}{ccc}
\hline \multirow{2}{*}{ Charge } & \multicolumn{2}{c}{ Composition } \\
\cline { 2 - 3 } & DMPC $^{+1}$ & DMPC \\
\hline 0 & 0 & 128 \\
+6 & 6 & 122 \\
+12 & 12 & 116 \\
\hline
\end{tabular}

Table S2. Pre-equilibrated lipid bilayers of 25\% DMPA/DMPC mixture at different ionizations of PA. DMPA ${ }^{0}, \mathrm{DMPA}^{-1}$ and $\mathrm{DMPA}^{-2}$ stands for the double, single and unprotonated PA species, respectively.

\begin{tabular}{cccc}
\hline \multirow{2}{*}{ Charge } & \multicolumn{3}{c}{ Composition } \\
\cline { 2 - 4 } & DMPA $^{0}$ & DMPA $^{-1}$ & DMPA $^{-2}$ \\
\hline 0 & 32 & 0 & 0 \\
-8 & 24 & 8 & 0 \\
-16 & 16 & 16 & 0 \\
-24 & 8 & 24 & 0 \\
-32 & 0 & 32 & 0 \\
-40 & 0 & 24 & 8 \\
-48 & 0 & 16 & 16 \\
-56 & 0 & 8 & 24 \\
-64 & 0 & 0 & 32 \\
\hline
\end{tabular}


Table S3. Equilibration and production times in the CpHMD-L simulations.

\begin{tabular}{cccc}
\multirow{2}{*}{ Membrane } & $\mathbf{p H}$ & \multicolumn{2}{c}{ Time Segment $(\boldsymbol{n s})$} \\
\cline { 3 - 4 } PC & & Equilibration & Production \\
\hline \multirow{3}{*}{ PC } & 0.0 & $0-10$ & $10-100$ \\
& 1.0 & $0-10$ & $10-100$ \\
& 2.0 & $0-10$ & $10-100$ \\
\hline & 0.0 & $0-80$ & $80-150$ \\
& 1.0 & $0-50$ & $50-120$ \\
& 1.5 & $0-40$ & $40-110$ \\
& 2.0 & $0-10$ & $10-100$ \\
& 3.0 & $0-10$ & $10-100$ \\
& 4.0 & $0-10$ & $10-100$ \\
& 5.0 & $0-10$ & $10-100$ \\
& 6.0 & $0-10$ & $10-100$ \\
& 7.0 & $0-10$ & $10-100$ \\
& 8.0 & $0-10$ & $10-100$ \\
& 9.0 & $0-10$ & $10-100$ \\
\hline
\end{tabular}

Table S4. Computed amount of $\mathrm{Na}^{+}$and $\mathrm{Cl}^{-}$ions for the various equilibration MD simulation systems at different molar fractions of PA/PC and different ionization values. "Predicted" refers to the amount of ions estimated by Poisson-Boltzmann that should be visible to the membrane, "Actual" refers to the number of ions added to the simulation, and "Evaluation" is the average number of ions explicitly seen by the membrane in the MD simulations.

\begin{tabular}{|c|c|c|c|c|c|c|c|c|}
\hline \multirow{2}{*}{$\mathrm{PA} / \mathrm{PC}(\%)$} & \multirow{2}{*}{ \# DMPA } & \multirow{2}{*}{ Charge } & \multicolumn{2}{|c|}{ Predicted } & \multicolumn{2}{|c|}{ Actual } & \multicolumn{2}{|c|}{ Evaluation } \\
\hline & & & $\mathrm{Na}^{+}$ & $\mathrm{Cl}^{-}$ & $\mathrm{Na}^{+}$ & $\mathrm{Cl}^{-}$ & $\mathrm{Na}^{+}$ & $\mathrm{Cl}^{-}$ \\
\hline \multirow{3}{*}{0.00} & \multirow{3}{*}{0} & 0 & 5.52 & 8.21 & 6 & 8 & 5.97 & 7.33 \\
\hline & & +6 & 5.31 & 8.52 & 5 & 8 & 4.93 & 7.38 \\
\hline & & +12 & 4.91 & 8.27 & 5 & 8 & 4.85 & 8.22 \\
\hline \multirow{9}{*}{25.00} & \multirow{9}{*}{32} & 0 & 4.60 & 6.37 & 5 & 7 & 5.00 & 6.32 \\
\hline & & -8 & 4.99 & 6.26 & 6 & 7 & 5.99 & 5.78 \\
\hline & & -16 & 5.86 & 6.75 & 6 & 7 & 5.99 & 6.31 \\
\hline & & -24 & 6.54 & 6.56 & 7 & 7 & 7.00 & 5.95 \\
\hline & & -32 & 7.24 & 6.57 & 8 & 7 & 7.99 & 6.06 \\
\hline & & -40 & 8.19 & 6.40 & 9 & 6 & 9.00 & 4.79 \\
\hline & & -48 & 9.21 & 6.35 & 9 & 6 & 9.00 & 5.11 \\
\hline & & -56 & 10.68 & 6.52 & 10 & 6 & 10.0 & 4.98 \\
\hline & & -64 & 11.89 & 6.36 & 12 & 6 & 12.0 & 4.60 \\
\hline
\end{tabular}


Table S5. Computed amount of $\mathrm{Na}^{+}$and $\mathrm{Cl}^{-}$ions for the various CpHMD-L simulation systems with a $25 \%$ molar fraction of $\mathrm{PA} / \mathrm{PC}$ at different $\mathrm{pH}$ values. "Predicted" refers to the amount of ions estimated by Poisson-Boltzmann that should be visible to the membrane, "Actual" refers to the number of ions added to the simulation, and "Evaluation" is the average number of ions explicitly seen by the membrane in the CpHMD-L simulations.

\begin{tabular}{|c|c|c|c|c|c|c|c|c|}
\hline \multirow{2}{*}{ System } & \multirow{2}{*}{$\mathrm{pH}$} & \multirow{2}{*}{ Charge } & \multicolumn{2}{|c|}{ Predicted } & \multicolumn{2}{|c|}{ Actual } & \multicolumn{2}{|c|}{ Evaluation } \\
\hline & & & $\mathrm{Na}^{+}$ & $\mathrm{Cl}^{-}$ & $\mathrm{Na}^{+}$ & $\mathrm{Cl}^{-}$ & $\mathrm{Na}^{+}$ & $\mathrm{Cl}^{-}$ \\
\hline \multirow{3}{*}{$\mathrm{PC}$} & 0.0 & $+8.73 \pm 1.15$ & 5.27 & 8.83 & 5 & 8 & 4.88 & 7.45 \\
\hline & 1.0 & $+2.53 \pm 0.46$ & 5.42 & 8.24 & 6 & 8 & 5.91 & 7.31 \\
\hline & 2.0 & $+0.42 \pm 0.10$ & 5.50 & 8.11 & 6 & 8 & 5.94 & 7.22 \\
\hline \multirow{11}{*}{$\mathrm{PA} / \mathrm{PC}$} & 0.0 & $+12.75 \pm 0.82$ & 4.83 & 7.84 & 5 & 7 & 4.93 & 6.75 \\
\hline & 1.0 & $-0.35 \pm 1.38$ & 5.05 & 7.37 & 5 & 7 & 4.96 & 6.69 \\
\hline & 1.5 & $-5.21 \pm 1.04$ & 5.24 & 7.19 & 6 & 7 & 5.94 & 6.17 \\
\hline & 2.0 & $-11.21 \pm 1.35$ & 5.87 & 7.10 & 6 & 7 & 6.00 & 6.50 \\
\hline & 3.0 & $-19.00 \pm 0.55$ & 6.22 & 7.14 & 7 & 7 & 6.99 & 6.17 \\
\hline & 4.0 & $-28.02 \pm 0.69$ & 7.18 & 6.77 & 8 & 7 & 7.99 & 6.11 \\
\hline & 5.0 & $-31.60 \pm 0.66$ & 7.24 & 6.85 & 8 & 7 & 8.00 & 6.16 \\
\hline & 6.0 & $-36.34 \pm 0.46$ & 7.80 & 6.89 & 8 & 7 & 8.00 & 6.33 \\
\hline & 7.0 & $-46.71 \pm 0.57$ & 9.56 & 6.45 & 10 & 6 & 10.00 & 4.78 \\
\hline & 8.0 & $-57.91 \pm 0.48$ & 11.29 & 6.39 & 12 & 6 & 12.00 & 4.45 \\
\hline & 9.0 & $-62.79 \pm 0.31$ & 11.79 & 6.43 & 12 & 6 & 12.00 & 4.63 \\
\hline
\end{tabular}




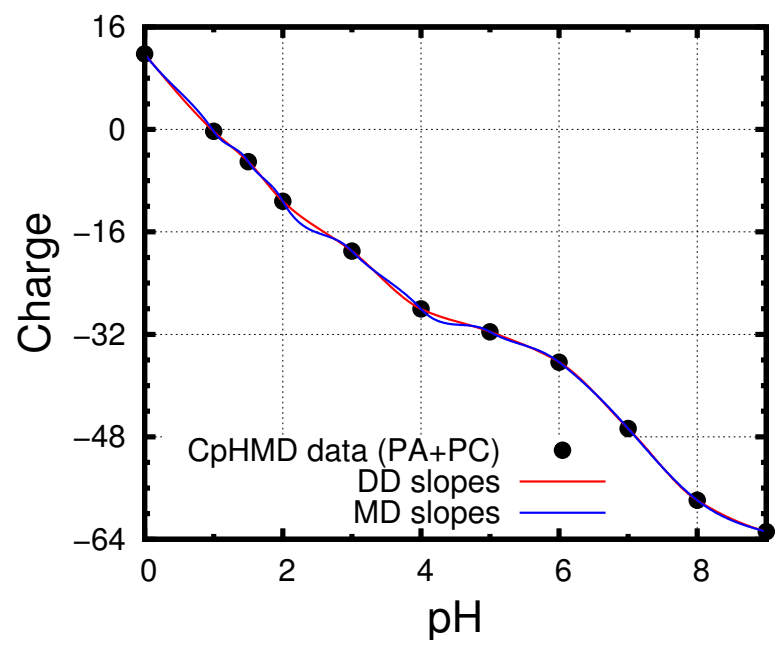

Figure S1. Simulated titration curves where the values are connected using cubic splines whose slopes are estimated using either the Davis-Dowden method (DD), or the relation $\partial \bar{Z} / \partial p H=-\ln (10) \sigma^{2}(\mathrm{MM})$, where $\bar{Z}$ and $\sigma$ are, respectively, the average and the standard deviation of the membrane charge at a given $\mathrm{pH}$ value. For more details, please see J Chem Theory Comput, 2015, 11, 2367. 


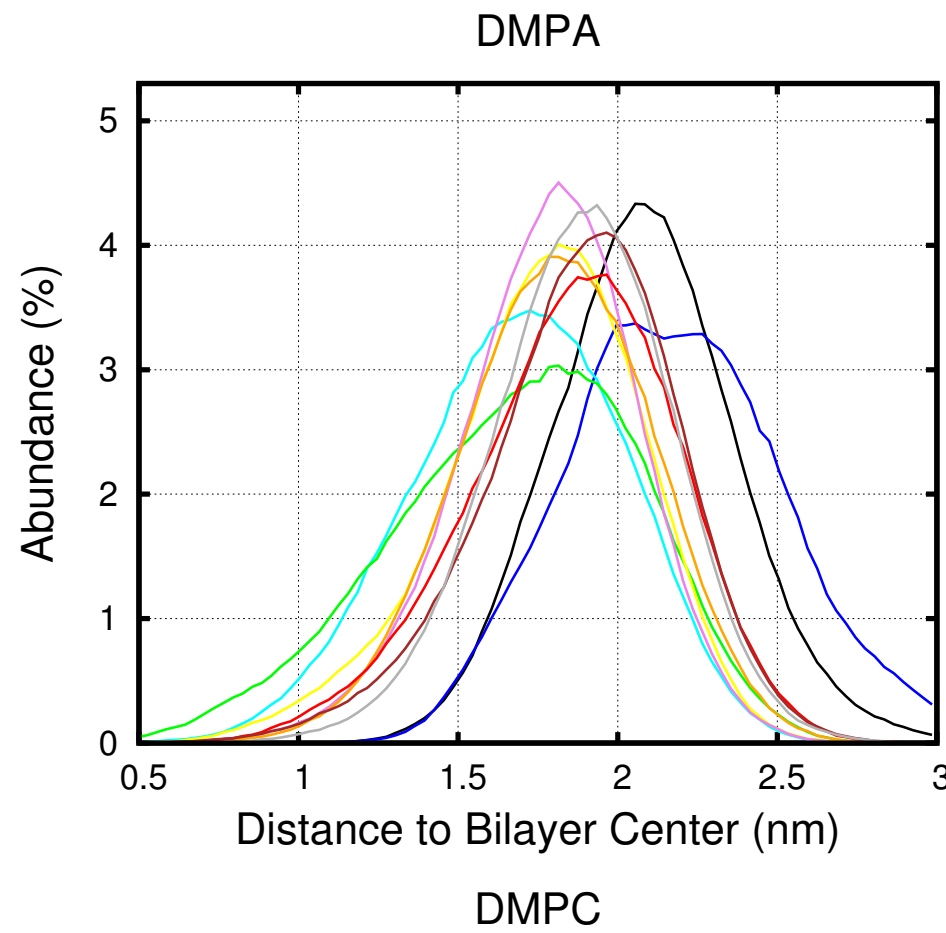

$\mathrm{pH} 0.0$

$\mathrm{pH} 1.0$

pH 2.0

pH 3.0

$\mathrm{pH} 4.0$

$\mathrm{pH} 5.0$

$\mathrm{pH} 6.0$

pH 7.0

$\mathrm{pH} 8.0$

pH 9.0

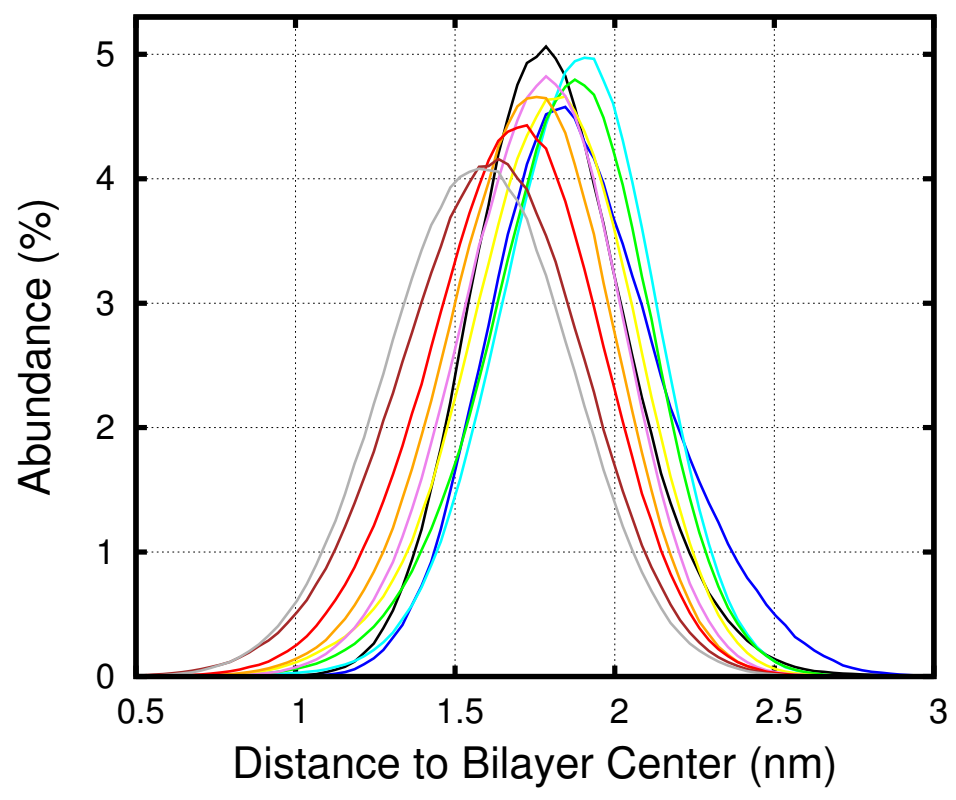

$\mathrm{pH} 0.0$

$\mathrm{pH} 1.0$

pH 2.0

pH 3.0

$\mathrm{pH} 4.0$

$\mathrm{pH} 5.0$

pH 6.0

$\mathrm{pH} 7.0$

pH 8.0

pH 9.0

Figure S2. Distributions of phosphor atoms distances to the center of the bilayer in the $25 \%$ DMPA/DMPC mixture at different $\mathrm{pH}$ values. The PA histograms are represented in the top graphic, while $\mathrm{PC}$ is in the bottom. The center of the bilayer is defined as the geometrical center (in the $z$-coordinate) of all 128 phosphor atoms. 


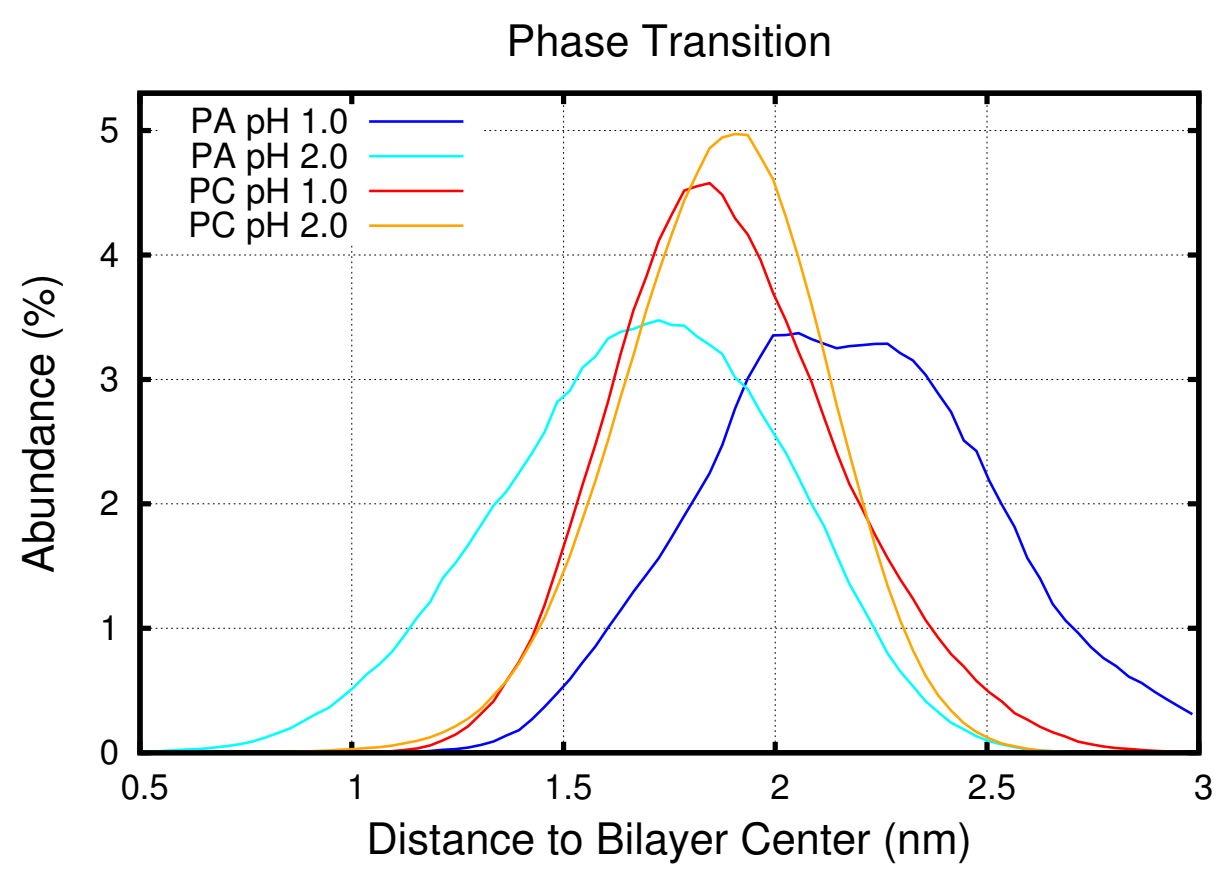

Figure S3. Distributions of phosphor atoms distances to the center of the bilayer in the $25 \%$ DMPA/DMPC mixture for $\mathrm{pH}$ values 1.0 and 2.0. The center of the bilayer is defined as the geometrical center (in the $z$-coordinate) of all 128 phosphor atoms.

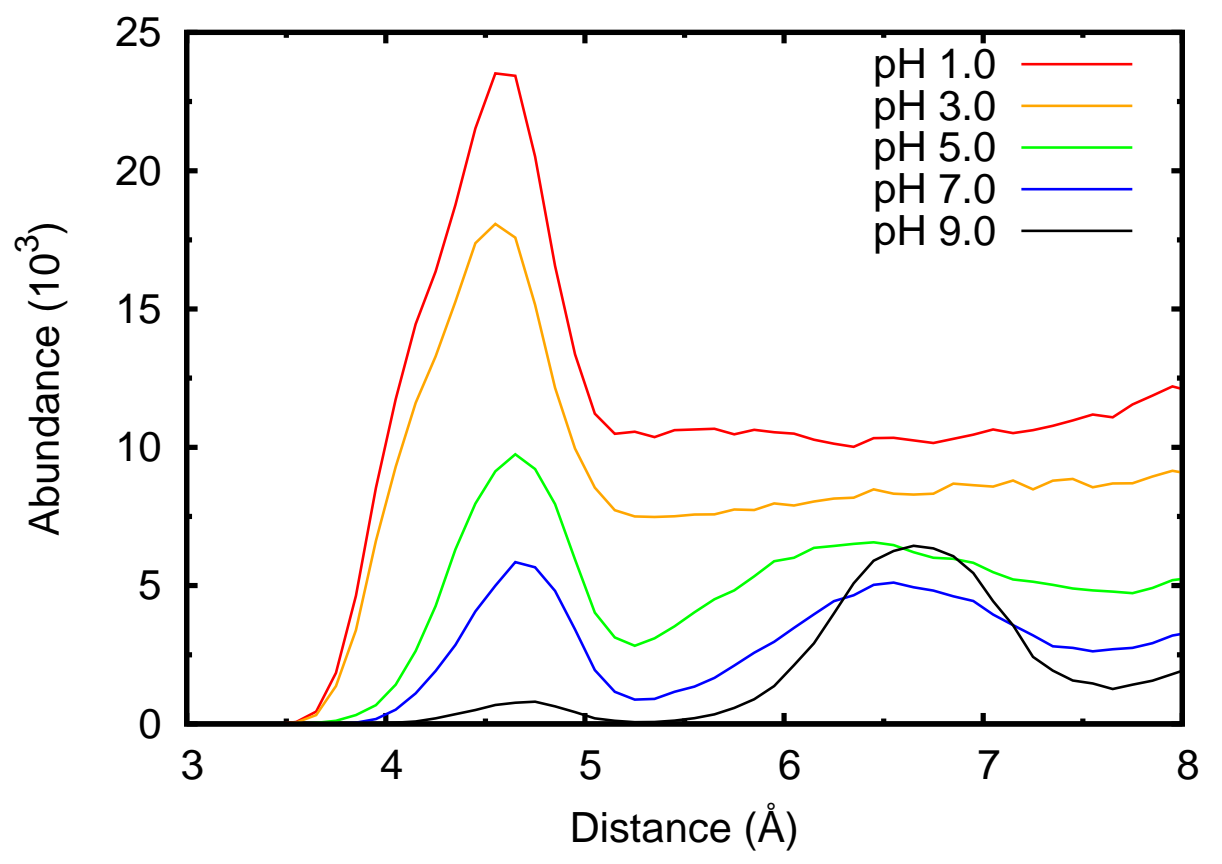

Figure S4. Distance histogram of DMPA in the 25\% DMPA/DMPC mixture at different $\mathrm{pH}$ values. From these curves, we chose the value $5.5 \AA$ as a cutoff to identify PA aggregation. 